EPJ Web of Conferences 52, 06002 (2013)

DOI: 10.1051/epjconf/20135206002

(C) Owned by the authors, published by EDP Sciences, 2013

\title{
Results from the Telescope Array and review of HiRes
}

\author{
P. Sokolsky ${ }^{1}$ \\ ${ }^{1}$ Department of Physics and Astronomy, University of Utah, Salt Lake City, Utah, USA, for the TA and HiRes \\ collaborations
}

\begin{abstract}
A new measurement of the cosmic ray spectrum using the Telescope Array surface arrays is presented. Results on the composition of ultra-high energy cosmic rays from air-fluorescence measurements of shower maximum distributions are presented. Preliminary data from the Telescope Array experiment are compared with HiRes measurements as well as Pierre Auger Observatory results. Systematic errors are discussed. Preliminary results on large scale anisotropy are shown.
\end{abstract}

\section{Introduction}

Structure in the cosmic ray spectrum above the "knee" $\left(10^{15} \mathrm{eV}\right)$ has been evident since the original Volcano Ranch experiment showed a slight hardening of the spectrum above $10^{19} \mathrm{eV}$. The Akeno, Yakutsk, Haverah Park, and Fly's Eye experiments all observed an "ankle" structure near $3 \times 10^{18} \mathrm{eV}$ with a hint of another break near $10^{17} \mathrm{eV}$ dubbed the "second knee". The HiRes/MIA hybrid experiment had similar evidence. New results from Kascake/Grande indicate the presence of a steepening in the heavy component of the spectrum near $10^{17} \mathrm{eV}$ as well [1]. Contemporary experiments such as HiRes[2], the Pierre Auger Observatory (PAO) [3] and Telescope Array (TA) [4] all observe clear evidence of an ankle structure followed by a cut-off near $6 \times 10^{19} \mathrm{eV}$ consistent with the predicted GZK steepening. The nature of these structures presents a challenge to theoretical understanding. Here we describe results from the TA surface detector on the cosmic ray spectrum above $10^{18}$ $\mathrm{eV}$, as well as preliminary results on composition using TA stereo data. We compare these results with previously published HiRes data and show that they are in good agreement. Comparison with Pierre Auger Observatory (PAO) results is not straightforward because of the different acceptances and analysis methods of the various experiments. Our current understanding of the systematics related to this is discussed.

\section{The Telescope Array (TA) Experiment}

The TA experiment is a hybrid experiment combining three air-fluorescence (FD) stations and a $762 \mathrm{~km}^{2}$ surface array (SD) composed of plastic scintillation counters with a $1.2 \mathrm{~km}$ spacing [4]. The air-fluorescence stations overlook the ground array and are spaced $\sim 35$ $\mathrm{km}$ apart. While two of the air-fluorescence stations (Black Rock and Long Ridge) are of a new optical and electronics design, the third ( Middle Drum ) is identical to the original HiRes I detector, except that its field of view extends to higher elevation angles (similar to HiRes II ). The existence of this detector allows us to directly compare event energies determined by the new fluorescence stations with the HiRes energy scale .

Events that trigger the fluorescence detectors can be reconstructed monocularly, in stereo, and in hybrid ( FD + SD ) mode. Monocular reconstruction has an angular resolution of $\sim 5$ degrees, while stereo and hybrid (fluorescence information plus surface array timing ) yields a resolution of $\sim .5$ degrees. Since geometrical reconstruction precision is important in composition studies, only stereo and hybrid data is used for this kind of analysis. The surface detector, consisting of 507 scintillation counters on $\mathrm{a} \sim 1.2 \mathrm{~km}$ grid, can be used in stand-alone mode to measure the energy and arrival direction of events. In this case, detector timing and pattern establishes the event geometry, while a fit to the EAS particle density profile produces a energy measurement. This energy determination is hadronic model dependent. However, energy found in this fashion can be compared with the fluorescence detector energy determination using hybrid events. Since the fluorescence method is largely model independent, we scale the surface detector energy to agree with the fluorescence energy scale to obtain the most accurate result. 


\section{Experimental Results}

\subsection{The TA surface detector spectrum}

The preliminary SD spectrum result is based on three years of data and 10,997 reconstructed events. Events with bad resolution are cut out of the data set. A novel part of this analysis is the use of extensive Monte Carlo simulations to establish the surface detector aperture. This allows us to extend the spectrum measurement well below the energy at which the aperture is saturated.

\section{Data/MC Comparisons}

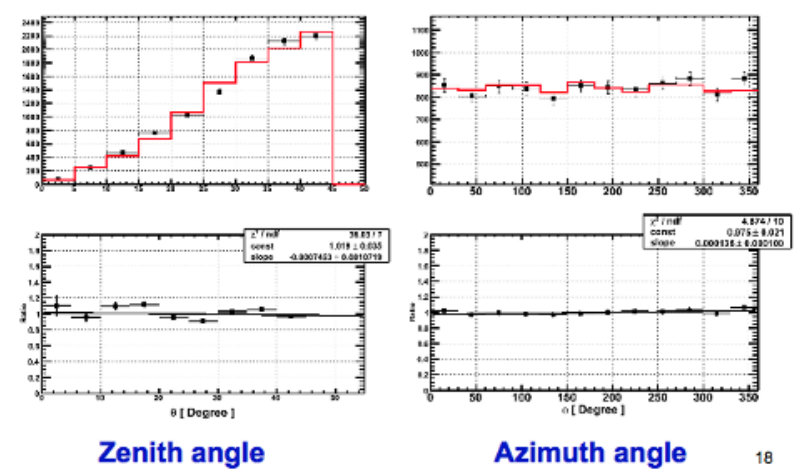

Fig. 1. Data and Monte Carlo comparisons for the SD zenith and azimuthal angles. Top plots show distributions, bottom plots are the ratios of data to simulations. Line is best fit to ratio.

\section{Data/MC Comparisons}

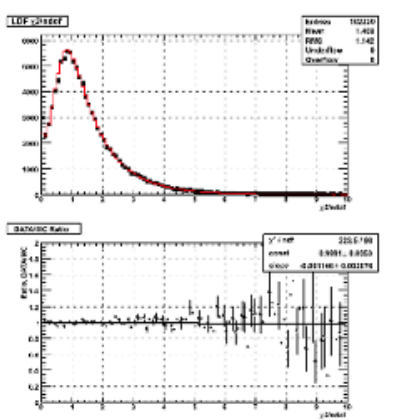

LDF $\mathrm{X}^{2} / \mathrm{dof}$

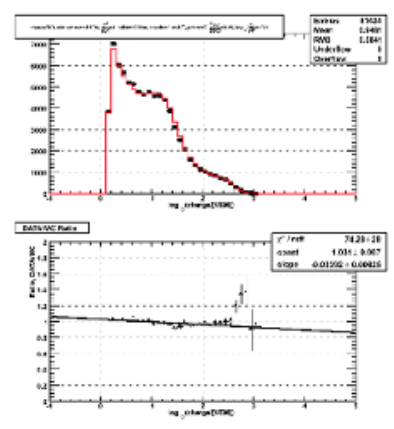

Counter pulse height
Fig. 2. Data and Monte Carlo comparisons for the goodness of fit of the SD lateral distribution function (left) and the measured and predicted SD detector pulse height (right).

The MC simulation uses the previously measured HiRes spectrum and composition as input. We use Corsika/QGSJet II in a new way to generate EAS without using the "thinning" approximation [5]. This is important to accurately reproduce particle fluctuations in the surface scintillation detectors. These events are thrown in an isotropic distribution and the trigger, front-end electronics and DAQ are accurately modeled. We then analyze the simulated data using the same reconstruction programs used for the real data. Various distributions comparing simulated to real raw data are examined to establish that we are accurately modeling the detector response to EAS.
Figs.1 and 2 shows some comparison distributions. All distributions show excellent agreement between data and MC.

Since the dependence of particle density on energy is zenith angle $(\Theta)$ dependent, a correction is introduced by constructing an energy table using QGSjet II proton events. A first estimate of event energy is done by interpolating between $\mathrm{S}(800)$ (event particle density at 800 meters $)$ and $\sec (\Theta)$ lines in this table. This method is clearly model dependent. However, a subset of SD data which also has fluorescence detector energy determination can be used to check the effect of the model dependence. Fig 3 show the relation between the $\mathrm{SD}$ and FD energies. While the relation is very linear, the SD energy is systematically too high relative to fluorescence by a factor of 1.27 . We therefore correct the SD energy scale by this factor in determining the spectrum. Note that the entire model dependence can be taken care of by the adjustment of a single parameter.

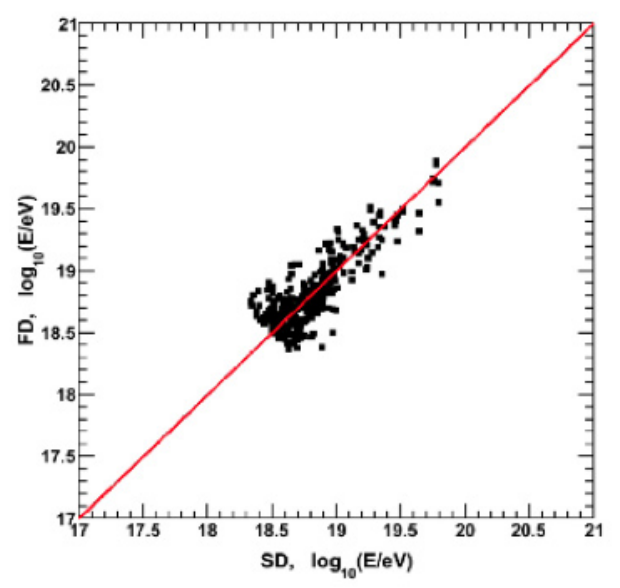

Fig. 3. Relation of FD and SD energy for a subset of hybrid events. SD energy has been rescaled down by a factor of 1.27 .

The MC data set is used to determine the SD acceptance ( see Fig 4 ). Because of the good agreement between data and $\mathrm{MC}$ in detector response, we can reliably determine the acceptance down to $\log E$ of 18.2. Fig. 5 shows the resultant spectrum. The ankle structure is observed clearly and a GZK-like cutoff is determined with a significance of 3.9 sigma. This spectrum is in excellent agreement with the previously published HiRes results. Good agreement with the PAO spectrum [3] is obtained after an $\sim 20 \%$ upward shift in the PAO energy scale. This shift is within the quoted systematic errors of the two experiments.

\subsection{Composition results from HiRes and TA}

HiRes stereo [7] and TA stereo [8] data have similar apertures and resolution in shower maximum (Xmax) near $10^{19} \mathrm{eV}$. It is therefore possible to compare the HiRes and TA elongation rates (mean Xmax vs energy) and Xmax fluctuation about the mean as a function of 
energy for both of them directly. Fig 6. shows the elongation rate for stereo HiRes and stereo TA. The results are in good agreement with each other as is the width of the Xmax fluctuation in energy bins (Fig. 7). We can thus combine the two data sets to increase the statistical power available. Fig 8 show the resultant elongation rate and Fig. 9 the fluctuations of Xmax about the mean for the combined data. The agreement between HiRes and TA data is remarkable given the different

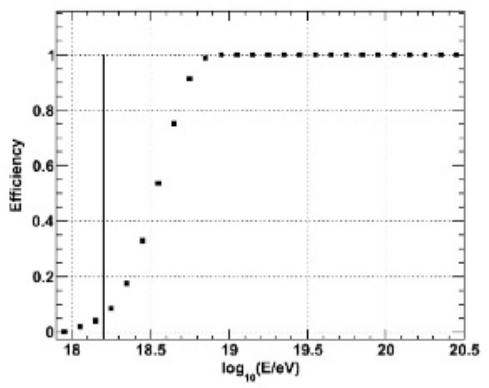

Fig. 4. SD aperture as a function of energy. Vertical bar indicates minimum energy for analysis.

\section{SD Energy Spectrum:}

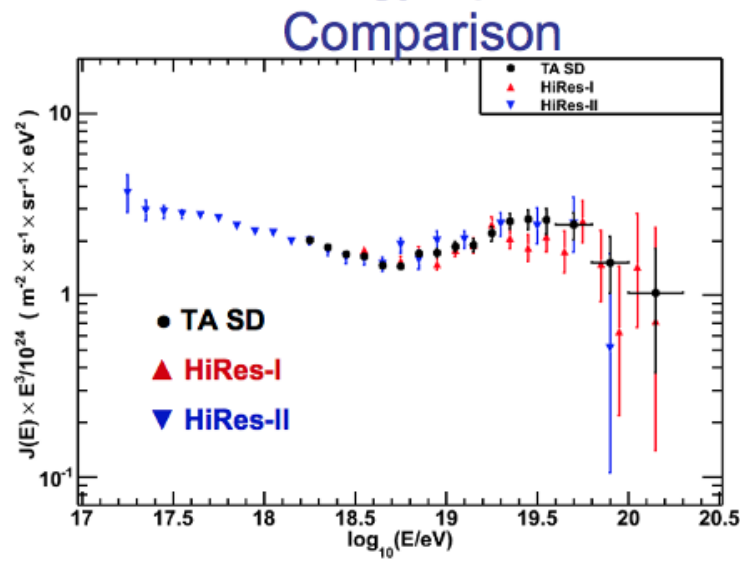

Fig. 5. TA SD spectrum and HiRes-I and HiRes-II published spectra.

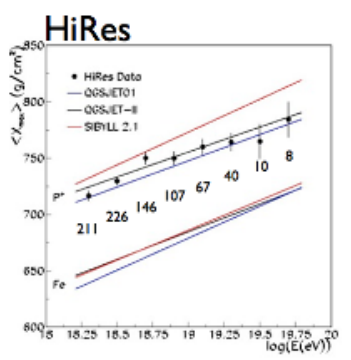

TA

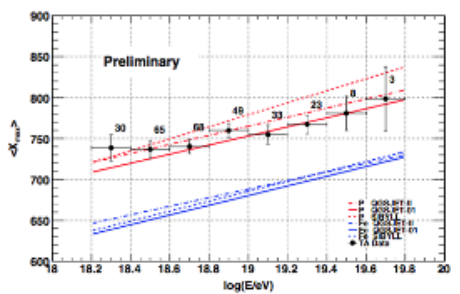

Fig. 6. HiRes and TA stereo elongation rates and model predictions. Model predictions have detector acceptance effects folded in.

detector design, potentially different atmospheric effects and completely different approach to reconstruction. Fig 10 shows the HiRes and TA elongation rates in comparison with hybrid PAO data [9]. While no acceptance corrections have been made, the elongation rate data from all three experiments is in excellent agreement. However, the Xmax fluctuations, as measured by HiRes and TA do not support the narrowing of the distribution widths at the highest energies as claimed by the PAO (see Fig. 11).

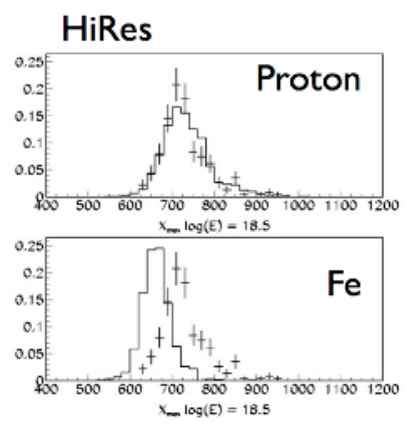

TA

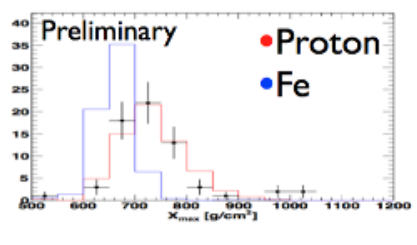

HiRes
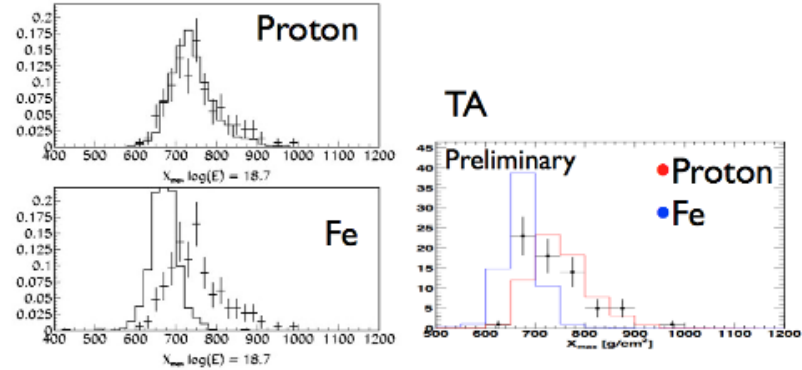

Fig. 7. HiRes and TA stereo Xmax distributions for $\log E 18.9$ and 19.1 energy bins and comparison to QGS Jet II proton and iron composition predictions (all detector effects included).

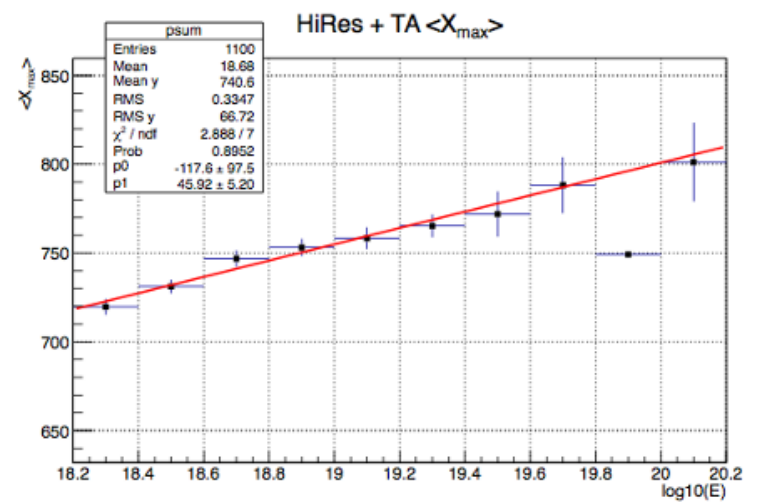

Fig. 8. Combined HiRes and TA stereo eleongation rate.

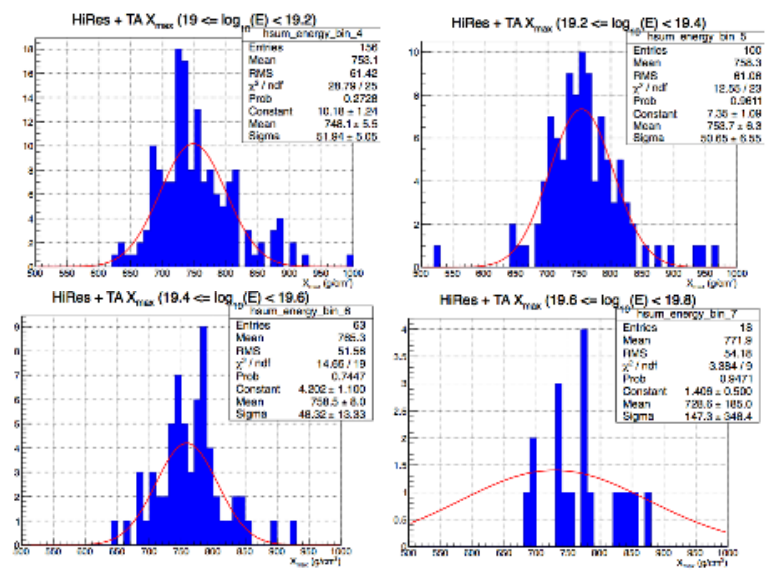

Fig. 9. Combined HiRes and TA stereo Xmax distributions Note that the predicted and measured Xmax resolution is $\sim 25$ $\mathrm{gm} / \mathrm{cm}^{2}$ for both experiments. 


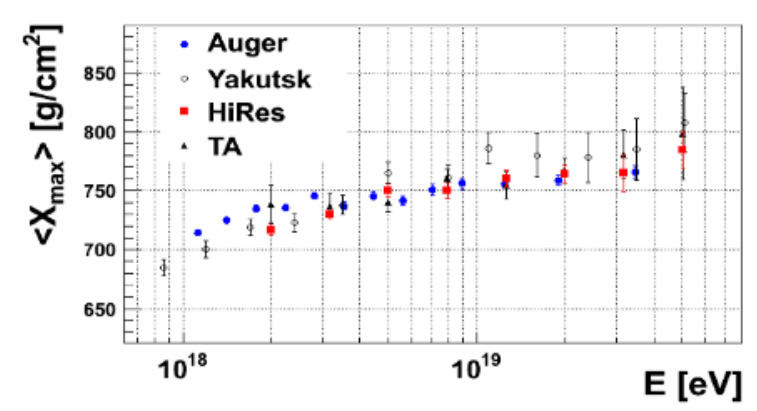

Fig. 10. Comparison of HiRes, TA and PAO elongation rates. No corrections for different detector acceptances between PAO and HiRes/TA have been made. The Yakutsk data is from a Cherenkov detector array which has a very different systematics.
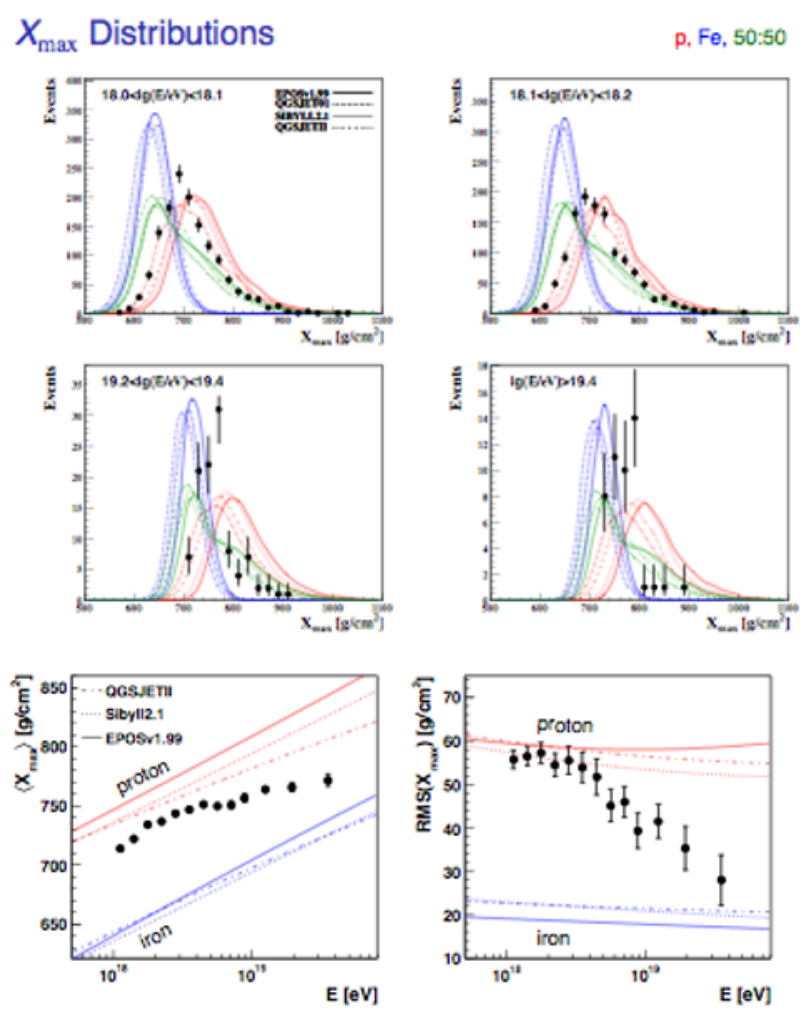

Fig. 11. PAO Xmax distributions, elongation rate and Xmax rms width as a function of energy.

\section{Interpretation of Xmax data}

While the HiRes and TA apertures are similar, the requirement for stereo triggering and good geometrical resolution introduces a small bias with respect to expectations for a purely protonic composition. Fig 12 shows that this bias, for the HiRes stereo data is $\sim 15$ $\mathrm{gm} / \mathrm{cm}^{2}$. The bias is negligible for a pure Fe composition. The PAO data is based on a hybrid analysis and uses a very different reconstruction approach. As a result, PAO data is reported to have minimal bias in elongation rate relative to pure protons. If this is correct, then the HiRes and TA elongation rates need to be shifted deeper by their bias, and the apparent agreement with PAO in elongation rate is thrown into disarray. In particular, HiRes and TA data is then in good agreement with a pure proton elongation rate (see Fig. 6), while PAO data requires a mixed composition at the highest energies ( see Fig 11 ). This, combined with the discrepancy in Xmax fluctuation width energy dependence makes the HiRes/TA and PAO results appear inconsistent.

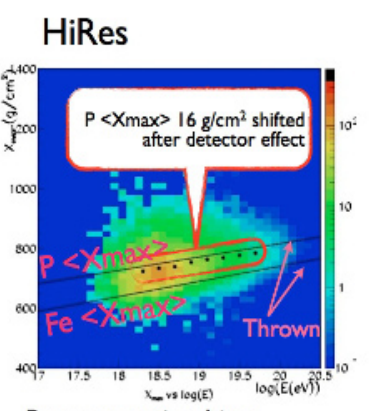

\section{Telescope Array}

$<$ Xmax $>$; Shower simulation

$$
\text { Reconstruction bias: }
$$

negligible w/ quality cut

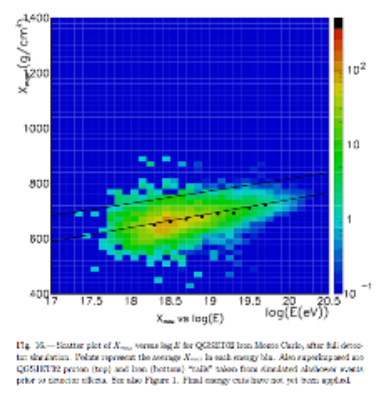

Fig. 12. Simulated proton and iron showers that trigger the HiRes stereo detectors. Lines indicate thrown elongation rate while points are the reconstructed elongation rate after detector acceptance and resolution.

It is surprising, however, that three separate experiments, with different biases in determining the elongation rate, would wind up with excellent agreement in the uncorrected data. Can this be telling us something important? Suppose, for example, that the actual cosmic ray flux is composed of protons, but that proton interactions at these energies produce showers that have Xmax shifted with respect to our current models. If the shift is to shallower depths, then the experimental bias in HiRes and TA becomes smaller. Somewhere between the predictions of current models for protons and for $\mathrm{Fe}$, the bias essentially vanishes. Similarly, if the actual cosmic ray flux was composed of a mixture of medium heavy nuclei such as Nitrogen and Carbon, our current models would produce an elongation rate which would lead to a much smaller bias in the HiRes and TA experiments. In other words, the bias in the elongation rate depends to some extent on the assumptions about the nature of the cosmic ray flux or the hadronic model used for protons. If we take the apparent agreement in uncorrected elongation rate seriously, then a range of compositions/hadronic models can be defined which would naturally lead to this consistency. Work to explore this is proceeding.

There remains the question of the Xmax fluctuation width differences. This is much less model dependent. The difference is not obviously due to reconstruction, since both experiments quote similar Xmax resolution. 
At current levels of statistics, it is not possible to say that HiRes/TA data are in complete contradiction to PAO widths at the highest energies, but the differences near $10^{19} \mathrm{eV}$ are becoming significant. Whether this is a residual systematic effect or the beginning of an emergence of a real North/South difference remains to be seen. Early hybrid data using the TA SD and the Middle Drum fluorescence detector again show good agreement with protons [10]. No obvious systematic differences between stereo and hybrid reconstruction methods have yet to emerge in the TA analysis.

\section{TA Anisotropy results}

TA SD data can be used to search for anisotropy in the cosmic ray arrival directions. We report on an analysis to study correlations of arrival directions with the local large scale structure (LLSS) [11]. We assume that cosmic rays are produced in a way that is proportional to the galactic density as measured by surveys such as the 2MASS $\mathrm{XSCz}$ catalog ( out to distances of $250 \mathrm{Mpc}$ ). For larger redshifts, we assume an isotropic distribution of cosmic ray sources. We assume an initial proton flux and take into account all propagation effects except magnetic fields. The effect of magnetic fields ( extragalactic and galactic) as well as finite angular resolution is roughly taken into account by a Gaussian smearing in arrival directions. The smearing angle is a parameter in the analysis. We then generate a predicted flux as a function of galactic coordinates and compare with the observed distribution using a flux sampling test. This procedure is done at three previously determined threshold energies, $10^{19}, 4 \times 10^{19}$ and $5.7 \times 10^{19} \mathrm{eV}$. Fig 13 shows the predicted fluxes and the observed event directions (note that detector acceptance is taken into account in these plots). Fig 14 shows the result of a K-S test relative to isotropy and the LLSS hypothesis. At the present level of significance, the data is consistent with both an isotropic origin and correlation with LLSS, except at the $10^{19} \mathrm{eV}$ threshold. Since a smearing angle is a poor representation of the effect of galactic fields at lower energies, we introduce a two-component galactic field model consistent with all existing measurements. Indeed, we find that this model makes the $10^{19} \mathrm{eV}$ threshold data compatible with the LLSS model for reasonable galactic parameters as long as a significant halo field is included.

\section{New projects}

There is now general agreement that a heavy to light transition occurs near the "second knee" though the exact energy scale is not clear. There is also agreement that the spectrum is light up to $10^{19} \mathrm{eV}$. Near the GZK cutoff, the experimental situation remains uncertain. TA and PAO proponents are now working together to resolve wether this is due to some poorly understood systematics or a real N/S hemispheric effect, perhaps reflecting differences in nearby cosmic ray sources.

\section{Data, and Models}
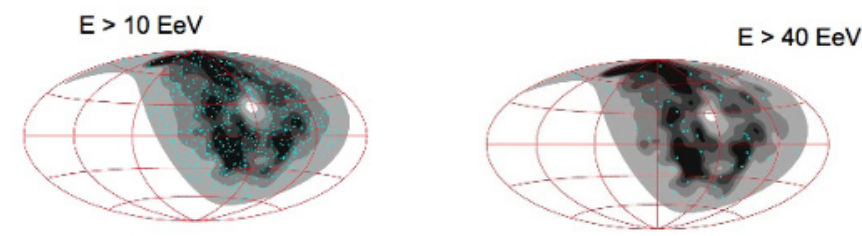

(smearing angle $=6^{\circ}$ )

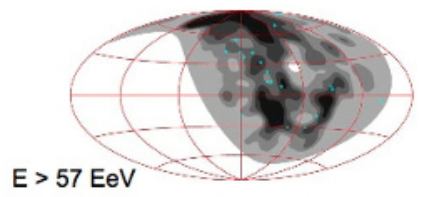

Fig. 13. Comparison of measured SD TA event arrival distributions with model LSS predictions for three energy thresholds.

\section{Results of K-S Test}

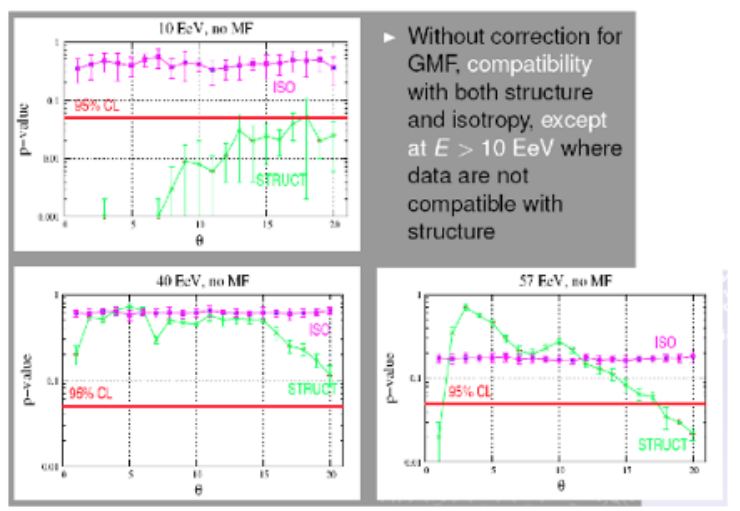

Fig. 14. KS test for three energy thresholds for isotropy and the LLSS model.

New experiments (TALE in the north and the PAO low energy extensions HEAT and AMIGA[12] in the South) will provide more precise information of the ankle and second knee composition issues. The TALE project (see Fig 15) will add an additional fluorescence station to the Middle Drum site to extend the detector viewing angles to $\sim 70$ degrees. This will be combined with a new infill array bridging the gap between the TA SD and the Middle Drum site. The intent is to study the spectrum and composition using the hybrid technique from $\log \mathrm{E}=16.5$ to 18.0. At the higher end, cross-calibration with the full TA detector will be possible and a single energy scale from the GZK on down will be established. Of particular interest will be the ability to measure EAS development at the same energy as the LHC. This will be the first time that a direct comparison between accelerator based measurements and cosmic ray EAS can be accomplished at ultra-high energies. 


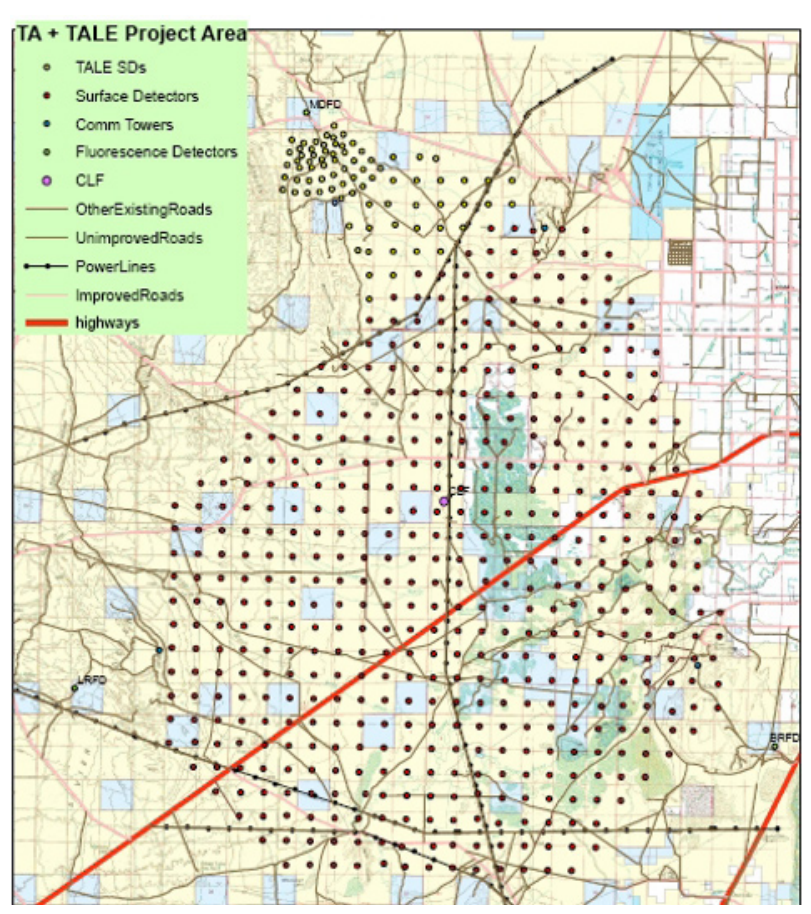

Fig. 15. TA and TALE arrays with fluorescence detection stations.

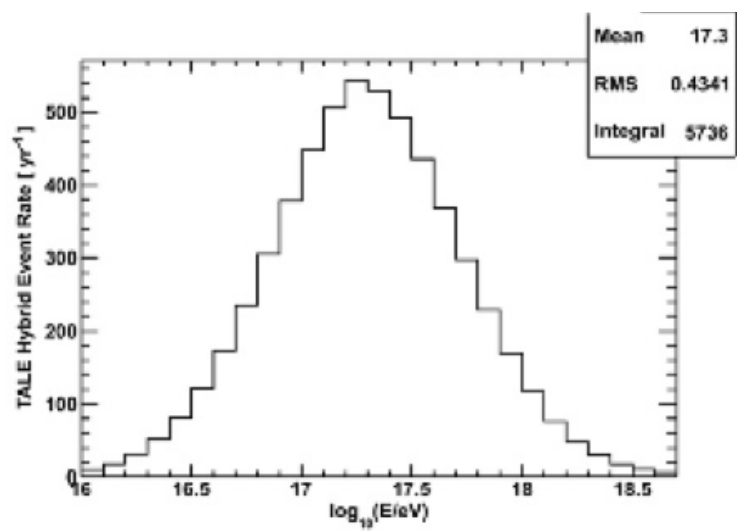

TALE hybrid events per year

Fig. 16. Expected energy distribution of hybrid events per year in the TALE detector.TA.

\section{References}

1. P. Sokolsky and G. Thomson, J. Phys. G, B43B, R401 (2007); M. Takeda et al., Phys. Rev. Lett. 81,1163 (1998); Astropart. Phys. 19,447 (2003).

2. R. U. Abbasi et al., Phys. Rev. Lett. 100, 101101 (2008)

3. J. Abraham et al., Phys. Rev. Lett. 101, 061101 (2008); Phys. Lett. 104, 091101 (2010).

4. T. Abbu-Zayyad et al., Nucl. Inst. Meth. A689, 87 (2012); H. Tokuno et al., Nucl. Inst. Meth. A676, 54 (2012); T. Abbu-Zayyad et al., Astropart. Phys. 39, 109 (2012)

5. B. T. Stokes et al., Astropart. Phys. 35,759 (2012)

6. T. Abbu-Zayyad et al., submitted to Ap. J.

7. R.U. Abbasi et al., Phys. Rev. Lett. 104161101 (2012)

8. Y. Tameda et al, in Proc. of $32^{\text {nd }}$ ICRC, Vol. 2, Beijing, 216 (2011)

9. J. Abraham et al., Phys. Rev. Lett. 104, 091101 (2008)

10. T. Abbu-Zayyed et al., in preparation

11. T. Abbu-Zayyad et al., Ap. J. 757, 26 (2012)

12. M.Platino et al., JINST 6, P08006 (2011); A. Etchegoyen et al., arXiv:0710.1646 astro-PH 